\title{
Achados neuropsicolinguísticos na síndrome de Crouzon:
}

\author{
relato de caso
}

\section{Neuropsycholinguistic findings in Crouzon syndrome: case report}

\author{
Juliana Fernandes Godoy ${ }^{1}$, Ana Carulina Pereira Spinardi ${ }^{2}$, Luis Gustavo Ducati ${ }^{3}$, Dagma Venturini Marques \\ Abramides $^{4}$, Mariza Ribeiro Feniman ${ }^{5}$, Adriano Yacubian-Fernandes ${ }^{6}$, Luciana Paula Maximino $^{7}$
}

\begin{abstract}
RESUMO
O objetivo do trabalho foi relatar um caso clínico sobre a síndrome de Crouzon, englobando as habilidades neuropsicolinguisticas. O estudo foi realizado com uma criança de oito anos e dois meses, do gênero masculino, com diagnóstico clínico da síndrome de Crouzon. Para a caracterização das habilidades foi realizada avaliação interdisciplinar com fonoaudiólogo, psicólogo e neurologista. A avaliação fonoaudiológica incluiu a avaliação das habilidades comunicativas, da linguagem oral, considerando as habilidades fonológicas, sintáticas, semânticas e pragmáticas, e da linguagem escrita. Para a avaliação neuropsicológica utilizou-se a escala Wechsler Scale for Children. A avaliação neurológica foi centrada no exame de ressonância magnética de encéfalo. O paciente também realizou avaliação audiológica, e apresentou perda auditiva condutiva de grau leve. Na avaliação clínica e formal da linguagem oral foi possível observar alterações nos aspectos fonológico e semântico da linguagem. Já com relação à linguagem escrita, observou-se desempenho aquém do esperado para a idade, caracterizado principalmente por alterações na escrita e na aritmética. Os resultados da avaliação neuropsicológica evidenciaram valores de Quociente Intelectual dentro dos padrões da normalidade. O resultado da ressonância magnética do encéfalo demonstrou alteração estrutural do sistema nervoso central. Pode-se concluir que os achados evidenciaram alterações nas habilidades de linguagem oral e escrita, além da presença de alteração estrutural do sistema nervoso central.
\end{abstract}

Descritores: Cognição; Comunicação; Linguagem; Craniossinostoses; Síndrome de Crouzon

\section{INTRODUÇÃO}

A síndrome de Crouzon (SC) é uma condição autossômica dominante, rara, também relacionada à idade paterna avançada, e causada por mutações no gene do receptor 2 do

Trabalho realizado no Ambulatório de Anomalias Craniofaciais do Hospital de Reabilitação de Anomalias Craniofaciais da Universidade de São Paulo USP - Bauru (SP), Brasil.

(1) Acadêmica do Curso de Fonoaudiologia da Faculdade de Odontologia de Bauru da Universidade de São Paulo - USP - Bauru (SP), Brasil.

(2) Pós-graduanda (Mestrado) em Fonoaudiologia da Faculdade de Odontologia de Bauru da Universidade de São Paulo - USP - Bauru (SP), Brasil.

(3) Mestre, Neurocirurgião do Centro Médico de Bauru - Bauru (SP), Brasil.

(4) Doutora, Professora do Departamento de Fonoaudiologia da Faculdade de Odontologia de Bauru da Universidade de São Paulo - USP - Bauru (SP), Brasil.

(5) Livre-docente, Professora do Departamento de Fonoaudiologia da Faculdade de Odontologia de Bauru da Universidade de São Paulo - USP - Bauru (SP), Brasil.

(6) Doutor, Professor do Departamento de Fonoaudiologia da Faculdade de Odontologia de Bauru da Universidade de São Paulo - USP - Bauru (SP), Brasil.

(7) Doutora, Professora do Departamento de Fonoaudiologia da Faculdade de Odontologia de Bauru da Universidade de São Paulo - USP - Bauru (SP), Brasil.

Endereço para correspondência: Luciana Paula Maximino. Al. Dr. Octávio Pinheiro Brizola, 9/75, Vila Universitária, Bauru (SP), Brasil, CEP: 17012-

101. E-mail: lumaximino@uol.com.br

Recebido em: 8/2/2009; Aceito em: 8/4/2010 fator de crescimento dos fibroblastos $\left(\right.$ FGFR2) ${ }^{(1)}$. Foi pela primeira vez descrita pelo neurologista Louis Edouard Octave Crouzon, seis anos após a publicação clássica da síndrome de Apert $^{(2)}$. Esta afecção tem incidência entre 15,5 e 16,5 por milhão de nascimentos, perfazendo cerca de $4 \%$ dos casos de craniossinostoses ${ }^{(3)}$.

Os achados clínicos mais frequentes incluem sinostose da sutura coronal com envolvimento ocasional de outras suturas, braquicefalia, frontal proeminente, proptose, hipertelorismo, estrabismo, hipoplasia maxilar, prognatismo, atresia do meato auditivo externo, malformação de Chiari e deficiência intelectual $^{(4)}$. Tal qual a síndrome de Apert, também apresenta variações significativas em seu espectro fenotípico, inclusive com relatos de manifestações cardíacas ${ }^{(5)}$.

São poucos os estudos publicados na literatura pertinente, relacionados aos aspectos cognitivos e de linguagem na SC. Poucas evidências sugerem que existe uma alta frequência de perda auditiva nesta síndrome ${ }^{(6)}$ e o mesmo pode ser inferido quanto aos aspectos cognitivos ${ }^{(7)}$.

Especificamente em relação à linguagem também poucos relatos são evidenciados; estudo anterior ${ }^{(8)}$ avaliou seis pacientes com craniossinostoses sindrômicas (quatro Apert, um Crouzon e um Saethre-Chotzen), utilizando o ITPA (Illinois Test of Psycholinguistic Abilities), o TVIP (Teste de Vocabulário por Imagens Peabody) e o DAS (Developmental 
Sentence Analysis). Todos os pacientes apresentaram alterações articulatórias, e cinco deles demonstraram alterações nas habilidades sintáticas e semânticas, tanto do ponto de vista expressivo quanto receptivo. Os níveis intelectuais variaram desde a normalidade até a deficiência intelectual de graus variados. Os autores sugeriram que, devido à alta prevalência de distúrbios fonoaudiológicos nos casos de SC, a intervenção deve ser precoce.

Analisando o desenvolvimento neuropsicológico dos pacientes com SC, alguns fatores foram postulados na literatura como capazes de influenciá-lo, dentre os quais hipertensão intra craniana (HIC), as alterações morfológicas encefálicas e a estimulação da criança.

Existe consenso na literatura no sentido de que a idade precoce no momento da cirurgia é fator relevante associado ao desenvolvimento mental (e, consequentemente, da linguagem e qualidade de vida) nas craniossinostoses sindrômicas ${ }^{(9)}$, por promover um alívio da HIC.

A SC, portanto, representa, dentre as anomalias craniofaciais, um contingente significativo de patologias que trarão, durante o desenvolvimento do indivíduo, agravos em diferentes funções do sistema nervoso central, seja envolvendo a cognição, seja envolvendo outras funções, como a linguagem. Desta forma, delinear o fenótipo desenvolvimental desses indivíduos é de fundamental importância no que tange à reabilitação de indivíduos com a SC. Portanto, o objetivo deste estudo foi investigar as habilidades neuropsicolinguísticas de um paciente com a SC.

\section{APRESENTAÇÃO DO CASO CLÍNICO}

O presente estudo foi realizado com uma criança do gênero masculino, com oito anos e dois meses de idade, encaminhado para avaliação interdisciplinar ao Hospital de Reabilitação de Anomalias Craniofaciais da Universidade de São Paulo de Bauru - HRAC de Bauru. A criança cursava a $2^{\mathrm{a}}$ série do ensino fundamental em escola pública.

A realização deste estudo, assim como a divulgação dos resultados, foram autorizadas pelo familiar responsável, por meio da assinatura do Termo de Consentimento Livre e Esclarecido. O estudo também obteve a aprovação do Comitê de Ética em Pesquisa do HRAC/USP, processo número 288/2006-SVAPEPE-CEP.

O fenótipo apresentado pela criança com a SC foi: palato alto e estrito, craniossinostose, fronte estreita, exoftalmia, fendas palpebrais oblíquas para baixo, nariz pequeno, hipoplasia do terço médio da face, prognatismo e orelhas proeminentes.

Para a avaliação neuropsicológica utilizou-se as escalas Wechsler Scale for Children em sua terceira edição (WISC-III). Os resultados foram apresentados sob forma de quociente de inteligência verbal (QIV), quociente de inteligência de execução (QIE) e quociente de inteligência total (QI). O valor de 70 foi utilizado como referência, sendo que resultado igual ou superior a 70 representa QI satisfatório, e menor do que 70, insatisfatório, (deficiência cognitiva), conforme preconizado pela Organização Mundial de Saúde (OMS) em 1992.

A avaliação audiológica constituiu-se de audiometria tonal liminar e imitanciometria.
Para a caracterização do perfil de comunicação da criança com SC foi realizada avaliação fonoaudiológica clínica que incluiu: avaliação das habilidades comunicativas, que analisou a intencionalidade e o uso de recursos comunicativos verbais e não-verbais utilizados; a linguagem oral, considerando as habilidades fonológicas, sintáticas, semânticas e pragmáticas, e a linguagem escrita. A criança foi avaliada por meio de conversa espontânea e semi-dirigida. A habilidade fonológica foi avaliada pela Avaliação Fonológica da Criança (AFC) para caracterização do inventário fonológico e dos processos fonológicos e do Perfil de Habilidades Fonológicas. Foi realizada a aplicação do Teste Illinois de Habilidades Psicolinguísticas ${ }^{(9)}$ (ITPA) com a finalidade de caracterizar o desempenho em tarefas viso-motoras e auditivo-vocais, incluindo aspectos linguísticos e memória. Este teste engloba 12 subtestes que avaliam a recepção auditiva e visual, a memória sequencial auditiva e visual, a associação auditiva e visual, a "closura" auditiva, visual e gramatical, a expressão verbal e manual e a combinação de sons. Para a avaliação do desenvolvimento da linguagem receptiva utilizou-se o TVIP. Os resultados foram expressos em pontos e classificados de acordo com as categorias descritas no teste: alto superior, alto inferior, médio alto, médio, médio inferior, baixo superior e baixo inferior. $\mathrm{O}$ Token Test foi utilizado para mensurar a capacidade de compreensão no nível receptivo do paciente; apresenta 20 símbolos, diferentes em forma, tamanho e cor e permite quantificar as respostas por meio da contagem de pontos, fornecendo um escore bruto, que será ajustado ao escore corrigido segundo a escolaridade e, posteriormente classificado em níveis de déficit de compreensão (média superior, média, média inferior ou normal, leve, moderado, grave e muito grave).

A avaliação clínica da linguagem escrita foi realizada por meio da solicitação de escrita livre de texto e complementada pela avaliação padronizada Teste de Desempenho Escolar (TDE). O TDE foi utilizado para avaliar as habilidades escolares do paciente, mais especificamente a escrita, a leitura e a aritmética. Os resultados obtidos foram classificados a partir de escores, segundo tabelas próprias propostas pelo manual, em: inferior, média e média superior.

$\mathrm{Na}$ avaliação por neuroimagem o paciente foi submetido ao exame de ressonância magnética do encéfalo nas sequências axial, coronal e sagital.

No que se refere à avaliação neuropsicológica, o Quadro 1 mostra os valores obtidos para o teste de Quociente Intelectual (QI) no paciente deste estudo. Os resultados evidenciaram valores de QI dentro dos padrões da normalidade.

Quadro 1. Achados da avaliação psicológica

\begin{tabular}{|l|c|c|c|}
\hline Idade & QIV & QIE & QIT \\
\hline 8 & 72 & 96 & 82 \\
\hline
\end{tabular}

Legenda: QIV = quociente intelectual verbal; $\mathrm{QIE}=$ quociente intelectual de execução; QIT = quociente intelectual total

Em relação à avaliação auditiva o paciente apresentou perda auditiva condutiva de grau leve em ambas às orelhas. Os testes de fala foram compatíveis com os limiares auditivos e a imitanciometria revelou curva timpanométrica tipo B e ausência de reflexos estapedianos ipsilaterais em ambas as orelhas. 
Quadro 2. Resultados obtidos nos testes

\begin{tabular}{|c|c|c|c|c|c|c|c|c|c|c|c|}
\hline \multicolumn{2}{|c|}{ Token Test } & \multicolumn{2}{|r|}{ TVIP } & \multicolumn{2}{|c|}{ TDE } & \multicolumn{6}{|c|}{ ITPA } \\
\hline Pont. & Class. & Pont. & Class. & Pont. & Class. & $A A$ & $\mathrm{AV}$ & CG & $\mathrm{CV}$ & MSA & MSV \\
\hline 28,76 & Leve & 72 & Baixa superior & 71 & Inferior & 32 & 37 & 38 & 35 & 26 & 32 \\
\hline
\end{tabular}

Legenda: TVIP = Teste por imagens Peabody; TDE = Teste de desempenho escolar; ITPA= Teste Illinois de habilidades psicolinguísticas; AA= associação auditiva; $\mathrm{AV}=$ associação visual; $\mathrm{CG}=$ closura visual; $\mathrm{CV}=$ closura gramatical; $\mathrm{MSA}=$ memória sequencial auditiva; $\mathrm{MSV}=$ memória sequencial visual; Pont = pontuação; Class. = classificação segundo o teste

Por meio da avaliação da linguagem oral foi possível observar alterações nos aspectos fonológico e semântico. Quanto ao aspecto fonológico foram observados os seguintes processos fonológicos não mais esperados para a idade: dessonorização de fricativas, simplificação de líquidas e de encontros consonantais. No que se refere a alteração semântica do ponto de vista expressivo, foi observado desvio por proximidade morfológica e fonológica, além de restrição de vocabulário receptivo, confirmado pelo TVIP.

Já com relação à linguagem escrita, observou-se desempenho aquém do esperado para a idade, caracterizado principalmente por alterações na escrita e aritmética. Na escrita foram observadas representações múltiplas, apoio na oralidade, omissão de letras, acréscimo de letras, trocas na grafia de fonemas surdos e sonoros. Quanto à leitura esta se apresenta no nível escolar do paciente.

Os resultados dos testes e provas complementares estão descritos no Quadro 2.

Os achados da avaliação fonoaudiológica evidenciaram alterações de linguagem englobando tanto a modalidade oral quanto a escrita e perda auditiva condutiva de grau leve

O resultado da ressonância magnética do encéfalo demonstrou ventriculomegalia (Figura 1).

\section{DISCUSSÃO}

Os dados da avaliação audiológica indicaram perda auditiva condutiva de grau leve. Este achado corrobora pesquisas anteriores, que caracterizaram o quadro audiológico da SC com grande variedade de alterações, entre elas as de orelha média e as perdas auditivas ${ }^{(6,9,10)}$.

No que diz respeito ao desenvolvimento neuropsicológico, é descrita uma prevalência de deficiência intelectual que varia de 10 a $20 \%$ na $S^{(11)}$. No presente estudo, o paciente não apresentou esta alteração, estando condizente com o desenvolvimento cognitivo normal, encontrado na maioria dos casos.

A avaliação fonoaudiológica, englobando tanto os aspectos perceptivos quanto os da linguagem, evidenciou alterações relacionadas à recepção, as quais foram confirmadas pelos achados clínicos e formais. Ressalta-se que a alteração do aspecto fonológico, verificada pela presença de processos fonológicos não mais esperados para a idade, não foi confirmada pela avaliação das habilidades fonológicas (PHF). A literatura mostra que crianças que apresentam alterações fonológicas utilizam diferentes níveis de consciência fonológica ao refletirem sobre a estrutura fonológica de sua língua ${ }^{(12)}$.

Escassos são os estudos na literatura nacional e internacional sobre a linguagem de pacientes com a SC. De forma global, estes referem a predisposição de alterações englobando tais habilidades ${ }^{(7,9,10)}$.

Em relação às alterações de linguagem como parte de quadros mais abrangentes, usualmente englobando tanto a oralidade quanto a escrita, muitas vezes o QI tende a estar próximo dos valores da normalidade, sendo que o comprometimento encontra-se em áreas específicas da cognição, levando a um desempenho escolar pobre. Considera-se que a maioria das crianças com este perfil neuropsicológico apresentem conhecimento linguístico prévio (linguagem oral) ${ }^{(13)}$, o que torna tais distúrbios mais brandos e específicos do que os distúrbios de linguagem.

O achado da avaliação neurológica por meio da ressonância magnética evidenciou a presença de ventriculomegalia. Estima-se que 30 a $70 \%$ dos pacientes com SC apresentem algum grau de dilatação ventricular, as quais podem ocorrer por uma

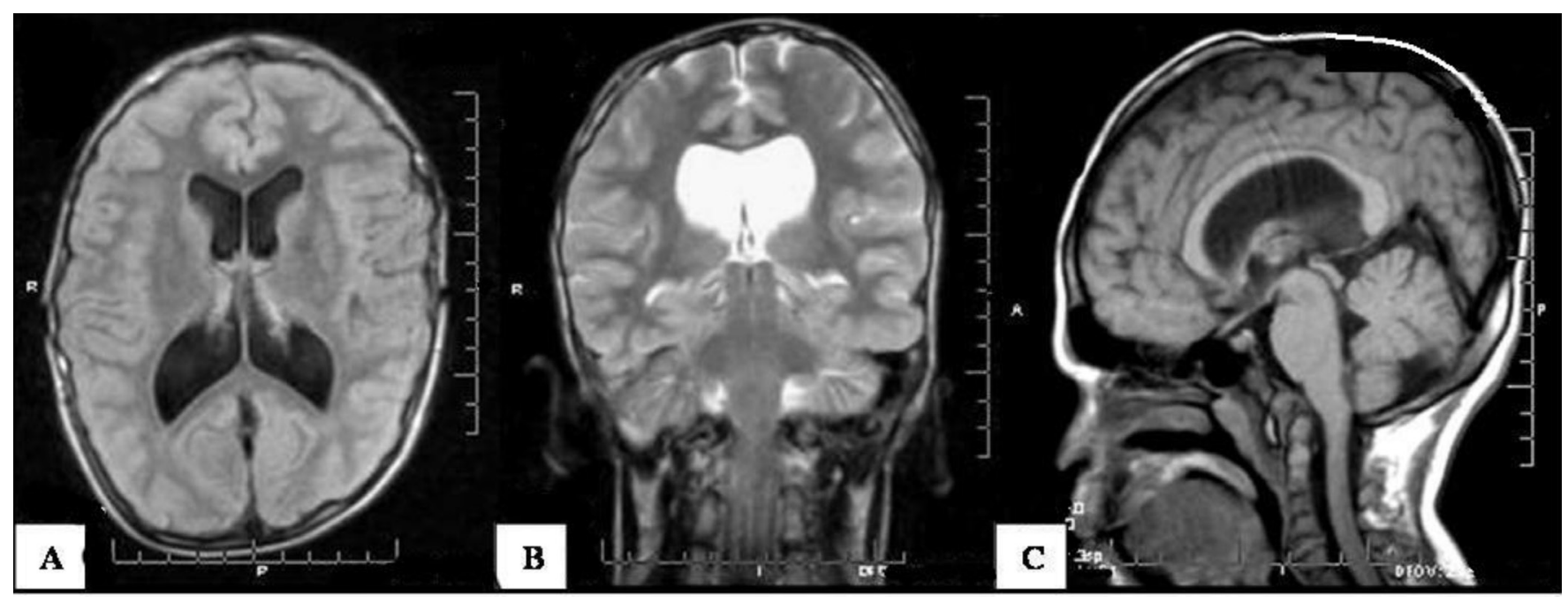

Figura 1. Ressonância magnética nas sequências axial $T 1(A)$, coronal $T 2$ (B) e sagital $T 1(C)$, evidenciando ventriculomegalia 
combinação dos seguintes mecanismos: resistência ao fluxo de líquor no nível da fossa posterior (fossa posterior pequena), e aumento da resistência à absorção liquórica nos seios venosos devido à hipertensão venosa existente nestes pacientes ${ }^{(14)}$. Os mesmos autores descrevem também que existe pouca evidência de associação entre ventriculomegalias não-hipertensivas e o prognóstico mental; ou seja, o desenvolvimento linguístico e cognitivo, com exceção dos quadros de hidrocefalia congênita severa, o que não se aplica ao presente estudo.

A hipótese do diagnóstico fonoaudiológico do caso, Distúrbio de Aprendizagem, refletiu as dificuldades no processamento das informações nos diferentes canais de aprendizagem expressas pelo paciente em questão, considerando que este termo foi utilizado como uma "expressão geral que se refere a um grupo heterogêneo de distúrbios, manifestados por dificuldades significativas na aquisição e no uso de capacidade de atenção, fala, leitura, escrita, raciocínio ou habilidades matemáticas. Esses distúrbios são intrínsecos ao individuo su- postamente devido a uma disfunção do sistema nervoso central e podem ocorrer ao longo da vida" de acordo com a definição do National Joint Comitee on Learning Disabilities ${ }^{(15)}$.

\section{CONCLUSÃO}

$\mathrm{O}$ indivíduo com SC avaliado neste estudo apresentou alterações nas habilidades de linguagem oral e escrita, além da presença de alteração estrutural do sistema nervoso central.

É importante considerar também que o paciente com a SC deve receber um acompanhamento multidisciplinar, o que lhe proporcionará diagnóstico precoce, intervenção adequada e desenvolvimento global satisfatório

Ressalta-se ainda a necessidade de estudos sistemáticos para a complementação dos achados, visando contribuir para o delineamento do fenótipo comportamental destas afecções genéticas.

\begin{abstract}
The aim of this study was to relate the neuropsycholinguistic abilities of a male child with eigth years and two months, diagnosed with Crouzon syndrome. The characterization of neropsycholinguitic abilities was based on a interdisciplinary evaluation carried out by a speech-language pathologist, a neurologist, and a psychologist. Speech-language evaluation included the assessment of communication skills, oral (phonological, syntactic, semantic and pragmatic abilities) and written language. The neuropsychological assessment used the Wechsler Scale for Children. The neurological evaluation was focused on the results of magnetic resonance imaging. The subject also carried out an audiological evaluation, which showed mild conductive hearing loss. In the oral language assessment, phonological and semantic deficits were observed. Written language performance was also below the expected for the subject's age, characterized mainly by writing and arithmetic deficits. The neuropsychological evaluation showed normal Intellectual Quotient. The results of the magnetic resonance imaging showed structural alterations of the central nervous system. Thus, the findings evidenced oral and written language deficits, and presence of structural alterations of the central nervous system.
\end{abstract}

Keywords: Cognition; Communication; Language; Craniosynostosis; Crouzon’s syndrome

\section{REFERÊNCIAS}

1. Glaser RL, Jiang W, Boyadjiev SA, Tran AK, Zachary AA, Van Maldergem L, et al. Paternal origin of FGFR2 mutations in sporadic cases of Crouzon syndrome and Pfeiffer syndrome. Am J Hum Genet. 2000;66(3):768-77.

2. Pilger TW. The cranio-facial hereditary syndrome of Crouzon. Int $\mathrm{J}$ Orthod. 1974;12(3):25-9.

3. Cohen MM Jr, Kreiborg S. Birth prevalence studies of the Crouzon syndrome: comparison of direct and indirect methods. Clin Genet. 1992;41(1):12-5.

4. Carinci F, Avantaggiato A, Curioni C. Crouzon syndrome: cephalometric analysis and evaluation of pathogenesis. Cleft Palate Craniofac J. 1994;31(3):201-9.

5. Schulz C, Kress W, Schömig A, Wessely R. Endocardial cushion defect in a patient with Crouzon syndrome carrying a mutation in the fibroblast growth factor receptor (FGFR)-2 gene. Clin Genet. 2007;72(4):305-7.

6. Orvidas LJ, Fabry LB, Diacova S, McDonald TJ. Hearing and otopathology in Crouzon syndrome. Laryngoscope. 1999;109(9):1372-5.

7. Yacubian-Fernandes A, Ducati LG, Silva MV, Abramides DVM, Perosa GB, Palhares A, et al. Síndrome de Crouzon: fatores envolvidos no desenvolvimento neuropsicológico e na qualidade de vida. Arq Neuropsiquiatr. 2007;65(2B):467-71.
8. Elfenbein JL, Waziri M, Morris HL. Verbal communication skills of six children with craniofacial anomalies. Cleft Palate J. 1981;18(1):59-64.

9. Arduino-Meirelles AP, Lacerda CBF, Gil-da-Silva-Lopes VL. Aspectos sobre desenvolvimento de linguagem oral em craniossinostoses sindrômicas. Pró-Fono. 2006;18(2): 213-20.

10. Gomes I, Limongi SCO, Neves SI, Couto MIV, Matas CG. Aspectos fonoaudiológicos na síndrome de Crouzon: estudo de caso. Rev CEFAC. 2008;10(3):303-10.

11. Noetzel MJ, Marsh JL, Palkes H, Gado M. Hydrocephalus and mental retardation in craniosynostosis. J Pediatr. 1985;107(6):885-92.

12. Lamprecht RR. Sobre os desvios fonológicos. In: Lamprecht RR, organizadora. Aquisição fonológica do português. Porto Alegre: Artmed; 2004. p 123-212.

13. Rotta NT, Guardiola A. Distúrbios de aprendizagem. In: Diament A, Cypel S. Neurologia infantil. 3a ed. São Paulo: Atheneu, 1996. p. 106274.

14. Collmann H, Sörensen N, Krauss J. Hydrocephalus in craniosynostosis: a review. Childs Nerv Syst. 2005;21(10):902 -12. Review.

15. Learning disabilities: issues on definition. National Joint Committee on Learning Disabilities. ASHA Suppl. 1991;(5):18-20. 